\title{
Associations between stroke lesion location and verbal fluency tests in a sub-acute stroke population
}

\author{
Ganesh M. Babulal \\ Department of Neurology; Washington University in St. Louis, Missouri, USA
}

\begin{abstract}
Background: In stroke hospitalizations, verbal fluency tests are used to evaluate executive abilities in order to assess the integrity of functioning and language. Prior studies show that clustering and switching during verbal fluency tasks are related to functioning of specific brain regions, such that they are thought to be more sensitive in detecting impairments in frontal regions compared to other brain regions.

Objective: Investigate the hypothesis that the stroke lesion location predicts both quantitative and qualitative (clustering and switching) aspects of fluency. Patients with frontal lesions will perform poorly on switching and phonemic tasks, while the patients with temporal lesions will perform poorly on clustering semantic tasks.

Method: Stroke inpatients ( $=99$ ) completed verbal fluency measures and structural brain scans within 72 hours of admission. Healthy controls (N=39) were recruited and administered verbal fluency measures. Fluency performance was evaluated by valid qualitative and quantitative analysis.

Results: Lesion location did not predict qualitative aspects of fluency and provided no support that frontal region lesions result in impairments in executive function. Hemispheric lesion location was not significant. Stroke patients preformed more poorly on fluency measures compared to controls.

Conclusion: Verbal fluency performance is affected by vascular brain damage resulting from stroke, but lesion location does not differentially affect qualitative or quantitative aspects of verbal fluency performance.
\end{abstract}

\section{Introduction}

In research and clinical settings, oral verbal fluency tasks are widely used to assess executive abilities in stroke populations [1,2]. Patients produce as many words as possible within a timeframe (typically one minute). Two types of fluency are assessed: phonemic (letter fluency) and semantic (category fluency) [3,4]. For phonemic fluency, patients are asked to produce as many words as possible beginning with a designated letter (e.g., F, A, S). For semantic fluency, patients produce as many words as possible belonging to a category (e.g., animals, food) [4]. Both tasks are thought to engage cognitive abilities that rely on different brain networks. Performance on phonemic fluency has been argued to reflect higher executive abilities supported largely by frontalregion functioning, while, performance on semantic fluency is thought to reflect memory processes supported largely by temporal-region functioning [1].

Imaging studies using positron emission tomography (PET) support the concept of region specialization during phonemic and semantic fluency tasks. Healthy adults have increased blood flow to the left frontal cortex when responding to letter prompts (e.g., S, B) and an increase in blood flow to the left temporal cortex was found when responding to categorical prompts (e.g., vegetables, clothes) [5]. Stimulus type may produce a more focal effect. There was greater blood flow in the anteromedial temporal and inferior parietal cortices for natural objects, but greater blood flow was observed in the left posterior temporal cortex for manmade objects. This association suggests a differentiated organization of the semantic representations in the brain, consistent with category-specific deficits of naming [4-6]. Participant characteristics may also influence performance (i.e., numbers of words generated) on verbal fluency tasks [7]. Age and education are associated with number of words generated, but not gender. Age and education adjusted norms are available $[4,8]$.

Fluency tasks typically utilize cut-offs or percentiles to detect problems in underlying cognitive processes $[1,8,9]$. Yet, studies suggest these scores may not comprehensively capture patient performance on fluency tasks; rather, errors types (e.g. perseveration) could give insight into impairments [10,11]. Troyer and colleagues [2,5] proposed that verbal fluency was a multifactorial process that may be further divided into two cognitive operations: clustering and switching, both of which can be assessed during fluency tasks to inform other aspects of performance. Clustering, or producing words within a phonemic or semantic category, is supported by temporal regions, whereas switching, or shifting between categories, is supported by frontal regions. Phonemic clusters are word groups that share a sound, either beginning with the same phoneme (e.g., farm, family), have a phonemic combination (e.g., froth, from), or end with a rhyme (e.g., stand, sand). Semantic clusters are words grouped by an aspect of meaning (e.g., cow, chicken, all from category farm animals). Within clusters, switching can occur in either semantic (cat, cheetah, monkey, lion) or phonemic tasks (stairs, stamps, stars, shops). Dual-task paradigms support a

Correspondence to: GM Babulal, Department of Neurology, Washington University School of Medicine, Campus Box 81114488 Forest Park, St. Louis, MO 63110, USA; Tel: (952) 334-8536; E-mail: babulalg@neuro.wustl.edu

Key words: verbal fluency, stroke, executive function, clustering, switching

Received: July 29, 2016; Accepted: August 20, 2016; Published: August 23, 2016 
clustering and switching distinction. Frontal region dependent-tasks (i.e., finger-tapping and producing words) interfere with switching, while temporal region dependent-tasks (i.e., object recognition) interfere with clustering $[7,12]$.

Some lesion studies do not support region specificity on fluency tasks, where primarily frontal damage impacts phonemic fluency and temporal damage affects semantic fluency. A meta-analysis of 31 fluency studies found focal frontal lesions produced impairments in both phonemic and semantic fluency with larger performance deficits in phonemic tasks [13]. In a study of patients with focal frontal lesions, there was no difference in the number of words produced between right and left patients; yet, the left frontal group produced more unusual animal names, whereas the right frontal group produced prototypical patterns of animal naming [14]. Another study of patients with focal lesions in frontal and temporal regions found small differences in switching and cluster sizes [12].

Small samples, lack of control groups and mixed-lesion groups used in prior studies limit the ability of verbal fluency tasks to accurately detect executive function impairments in stroke patients. Further, there is limited information on the relationship between lesions and fluency performance, specifically when examining the clustering and switching processes of executive function. The complexity of neuroanatomical and cognitive processes underlying clustering and switching operations requires further analysis to draw conclusions regarding fluency in stroke patients and related outcomes. In the current study, we examined a large sample of sub-acute stroke patients to determine if measures of verbal fluency are able to differentiate lesion location based on clustering and switching performance in phonemic and semantic fluency tasks. We hypothesize that frontal group will perform poorly on switching and phonemic tasks, while the temporal group would perform poorly on clustering semantic tasks.

\section{Methods}

Study population: Fluency data were collected during acute stroke hospital admissions at a major hospital in the United States of America, North America. This registry and methodology has been published [15]. Patients were excluded from the current study if there was a documented history of mental illness, evidence of alcohol or substance abuse, and dementia (Short Blessed Test $\leq 10$ ). Participants with severe aphasia were excluded because they were unable to complete the verbal fluency tasks. Participants were included if lesion descriptions with imaging and fluency data were available. Structural brain scans and verbal fluency data were obtained within 72 hours of hospital admission. Eligible controls were recruited and tested in a research laboratory at Washington University School of Medicine. All aspects of the study were approved by the Institutional Review Board.

Lesions were defined based on structural imaging from magnetic resonance imaging (MRI) or computerized tomography (CT) and classified according to vascular templates [16] and by clinical neuroradiological reports. Patients were grouped based on lesion location. Due to the broad nature of the lesion descriptions, dividing the participants into several highly specific lesion groups was unfeasible. Instead, patients were grouped into categories: frontal and non-frontal.

Study procedure Speech language pathologists administered fluency tasks as part of a larger cognitive-linguistic test battery. During the phonemic task, participants were instructed that they would have one minute to generate as many words as possible that began with specified letters (i.e., F,A,S). An example was provided along with sample responses to a letter prompt ('N') not used in the task. Participants were instructed not to respond with proper nouns (e.g., Nathan), words with a shared root but different endings (e.g., nap, napping) or numbers (e.g., nineteen). During the semantic fluency task, participants were instructed that they would have one minute to generate as many words as possible belonging to a specified category (i.e., animals).

Quantitative scoring To examine number of correct words, six types of responses were evaluated and scored. Numbers of correct words were recorded as non-repeated words, conforming to task directions. Perseverative errors were recorded when a word was repeated more than once during responding to one of the four prompts (i.e., F, A, $S$, animals). However, if participants produced "anteater" in response to the ' $A$ ' prompt, it was not counted as a perseverative error when produced again in response to the 'animal" prompt unless repeated twice in response to the "animal" prompt. Root-word variations depended on the context were not always counted as perseverative errors. For example, "fast, fasting" were scored as two correct answers. A similar variation such as "food, foods," however, was counted as a perseverative error. Intrusion errors were scored as words that did not fall into the category represented by the prompt. However, for phonemic fluency tests, misspellings were not counted as errors if they began with a phoneme appropriate to the letter being tested. For example, "phenomenon" was scored as correct in response to 'F." This exception was allowed to reduce the impact of spelling skill on performance. A special rule was made for proper noun errors. The instructions clearly state that proper nouns are incorrect responses. Participants' responses revealed that not all proper nouns are treated as such. For example, "French fries" contains a proper noun that was frequently given during letter naming. When scored, proper nouns such as these were counted as correct responses under the assumption that they are thought of as object names rather than proper nouns. Finally, diminutive variations are variations of the same word that do not share a common root-word. For example, "dog, puppy" was scored as two correct answers. Category names, if followed by specific examples, were diminutive variations and were counted as correct. For example, "dog, Dalmatian, golden retriever" was counted as three correct variations. The justification for this method of scoring is that diminutive variations result from an incomplete understanding of instructions. Since verbal fluency measures are being linked to lesions with the expectation of assessing impairments, ignoring diminutive variation responses would limit the aims of this study. Diminutive variations were counted in the number of correct responses.

Clustering and switching scoring Adjacent clusters sharing a word or words were counted as belonging to the cluster that seemed most logical or the cluster that resulted in a larger cluster size. For example, "rat, mouse, cat, dog" was scored as two clusters with one switch, where "cat" was counted in a cluster with "rat, mouse" and dog was counted as the switch. Consistent with this rule, clusters appearing within larger clusters were counted as members of the overarching cluster. For example, "rabbit, rhino, rat, rodent" was counted as a phonemic cluster of four items.

Phonemic clusters were scored differently for semantic and phonemic fluency tests. For animal naming, phonemic clusters were groups of responses that began with the same phoneme. For letter naming, since all correct responses began with the same phoneme, phonemic clusters were based on initial phoneme combinations or rhymes. For example, "food, forward" was not scored as a cluster because the words did not share an initial sound. However, "flee, fly," 
was scored as a phonemic cluster because the initial sound is the same in both words. For animal naming, semantic clusters were largely determined based on fit to published subcategories [2]. Commonly generated examples in subcategory lists were used for reference (S1).

National Institute of Health Stroke Scale (NIHSS) The NIHSS is a conventional measure of stroke severity in acute care settings [17]. The 15-item measure assesses level of consciousness, extraocular movements, visual fields, facial muscle function, extremity strength, sensory function, coordination (ataxia), language (aphasia), speech (dysarthria), and hemi-inattention (neglect) on a scale $0-4$, with 0 being normal or no impairment [18]. The NIHSS is commonly used as an initial assessment to inform acute care, rehabilitation and discharge; in addition, it is strongly associated with measures assessing the ability to complete activities of daily living [19].

Statistical analysis Following the scoring scheme proposed by Troyer and colleagues, results from both tasks were classified into five categories for analysis: correct responses, number of errors, number of switches, number of clusters and number of words in cluster $[6,7,12]$. The average number of responses from the three phonemic fluency trials $(\mathrm{F}, \mathrm{A}, \mathrm{S})$ and the total number of responses obtained from the semantic fluency task (animals) were calculated and used for analysis. Demographic characteristics were evaluated using nonparametric statistics. Group differences among controls and patients were evaluated by student's $t$-test and one-way ANOVAs with post-hoc tests. All statistical analyses were performed using SPSS version 21 (Chicago, IL, USA).

\section{Results}

The final sample included 138 participants with ages ranging 27-97 years (Table 1). There was a difference in gender but not age or education among the controls and stroke patients. In subgroup analyses of the stroke sample, there were no statistically significant difference in age, education, sex or NIHSS score among the frontal and non-frontal groups. In the total sample, there were 48 right hemisphere lesions, 25 left hemisphere lesions and 26 bilateral lesions. There were no statistically differences between the three groups on any of the demographic variables or the measures of verbal fluency.

Relationships between demographic, groups, and fluency measures Correlations between age, education and performance on phonemic (letter task) and semantic (animal task) fluency was calculated for the total sample (Table 2). Age was negatively correlated with number of correct responses on both letter and animal fluency tasks, such that number of correct responses decreased as age increased for both tasks. Age was positively correlated with errors on letter fluency and animal fluency tasks. Education was correlated with number of correct responses on letter fluency. Letter fluency switches and numbers of clusters were also correlated with education. When the stroke and control groups were analyzed separately, the relationship between age and education changed for each group (Table 2). Stroke group: age correlated with number correct and errors on letter fluency and cluster size on animal fluency. Education did not correlate with any measures of letter or animal fluency for the stroke group. Control group: age correlated with number correct and number of switches on both letter and animal fluency and errors on animal fluency. Education correlated with number correct on both letter and animal fluency and number of switches and number of clusters for letter fluency.

Phonemic fluency task To evaluate differences on the phonemic fluency task, we examined performance among frontal, non-frontal, and control groups. A one-way ANOVA revealed a significant effect of group on the number of correct responses $[\mathrm{F}(2,135)=62.2, p<.001]$. Post hoc Tukey HSD tests indicated that the control group $(\mathrm{M}=11.8$, $95 \%$ CI $[10.9,12.6])$ differed significantly from the frontal $(M=5.9$, $95 \% \mathrm{CI}[5.3,6.6])$ and non-frontal $(\mathrm{M}=6.2,95 \% \mathrm{CI}[5.2,7.1])$ groups, $p<.001$; there was no difference between the frontal and non-frontal

Table 1. Demographics of stroke and control groups.

\begin{tabular}{|c|c|c|c|c|c|c|}
\hline & $\begin{array}{c}\text { Controls } \\
(\mathrm{N}=39)\end{array}$ & $\begin{array}{l}\text { Stroke } \\
(\mathrm{N}=99)\end{array}$ & $p$ & $\begin{array}{l}\text { Frontal Group } \\
\quad(\mathbf{N}=56)\end{array}$ & $\begin{array}{l}\text { Non-Frontal Group } \\
(\mathbf{N}=\mathbf{4 3})\end{array}$ & $p$ \\
\hline $\begin{array}{l}\text { Age (Y) } \\
\text { Mean (SD) }\end{array}$ & $67.6(15.8)$ & 64.4 (13.9) & 0.452 & $63.0(14.3)$ & $66.2(13.2)$ & 0.486 \\
\hline $\begin{array}{l}\text { Education (Y) } \\
\text { Mean (SD) }\end{array}$ & $13.1(3.24)$ & $12.2(2.39)$ & 0.068 & $11.9(2.60)$ & $12.5(2.07)$ & 0.294 \\
\hline $\begin{array}{l}\text { Sex } \\
\text { Male } \\
\text { Female }\end{array}$ & $\begin{array}{c}9 \\
30\end{array}$ & $\begin{array}{l}52 \\
47\end{array}$ & 0.002 & $\begin{array}{l}30 \\
26\end{array}$ & $\begin{array}{l}22 \\
21\end{array}$ & 0.455 \\
\hline $\begin{array}{l}\text { NIHSS } \\
\text { Mean (SD) }\end{array}$ & - & $6.95(4.59)$ & - & $7.66(4.84)$ & $6.02(4.10)$ & 0.286 \\
\hline
\end{tabular}

Table 2. Age, education and fluency associations among stroke and control groups.

\begin{tabular}{|c|c|c|c|c|}
\hline \multirow[b]{2}{*}{ Variable } & \multicolumn{2}{|c|}{ Stroke } & \multicolumn{2}{|c|}{ Control } \\
\hline & Age (yrs) & Education (yrs) & Age (yrs) & Education (yrs) \\
\hline FAS Correct & $-0.23^{*}$ & 0.05 & $-0.403 * *$ & $0.567 * *$ \\
\hline FAS Errors & $.23 * *$ & -0.03 & -0.024 & -0.40 \\
\hline FAS Switches & -0.12 & -0.02 & $-0.422 * *$ & $.526^{* *}$ \\
\hline FAS Clusters & -0.16 & 0.18 & -0.109 & $0.302 *$ \\
\hline FAS Cluster Size & -0.14 & -0.13 & -0.141 & 0.039 \\
\hline Animal Correct & -0.16 & -0.03 & $-0.502 * *$ & $.355^{*}$ \\
\hline Animal Errors & 0.10 & 0.02 & $0.363 *$ & -0.265 \\
\hline Animal Switches & -0.04 & -0.08 & $-0.476 * *$ & .291 \\
\hline Animal Clusters & -0.10 & -0.03 & -0.046 & 0.035 \\
\hline Animal Cluster Size & $-0.24 *$ & 0.05 & -0.211 & 0.186 \\
\hline
\end{tabular}

$* * p<.001 ; * p<.05$. 
groups. Of particular interest was whether switching, number of words in clusters, and cluster size in letter fluency was different across three groups, as predicted by the literature. A one-way ANOVA indicated that all three dependent measures were statistically significant [switching: $\mathrm{F}(2,135)=53.0, p<.001$; words in a cluster: $\mathrm{F}(2,135)=32.9$, $p<.001$; cluster size: $\mathrm{F}(2,135)=17.5, p<.001]$. Post hoc comparisons showed that the control group differed significantly across switching $(\mathrm{M}=8.7895 \% \mathrm{CI}[8.0,9.5])$, words in a cluster $(\mathrm{M}=4.6,95 \% \mathrm{CI}[3.0$, 5.1]) and cluster size $(\mathrm{M}=2.4,95 \% \mathrm{CI}[2.2,2]$.$) from the frontal group$ (switching ( $\mathrm{M}=4.395 \% \mathrm{CI}[3.8,4.9])$; words in a cluster $(\mathrm{M}=1.9,95 \%$ CI $[1.6,2.3])$; cluster size $(\mathrm{M}=1.8,95 \% \mathrm{CI}[1.6,1.8]))$ and non-frontal group (switching $(\mathrm{M}=4.395 \% \mathrm{CI}[3.5,5.0])$; words in a cluster $(\mathrm{M}=$ $2.1,95 \%$ CI $[1.4,2.7])$; cluster size $(\mathrm{M}=1.8,95 \% \mathrm{CI}[1.5,1.9])), p<.001$, yet the frontal and non-frontal groups did not differ on any of the three measures of letter fluency.

Semantic fluency task To assess performance differences by group in semantic fluency, a one-way ANOVA revealed a significant effect on the number of correct response $[\mathrm{F}(2,135)=10.6, p<.001]$. Tukey HSD tests indicated that the control group $(\mathrm{M}=15.0,95 \% \mathrm{CI}[13.6,16.3])$ differed significantly from the frontal $(\mathrm{M}=10.9,95 \% \mathrm{CI}[9.8,12.1])$ and non-frontal groups $(\mathrm{M}=11.8,95 \% \mathrm{CI}[10.3,13.2]), p<.001$ while the two stroke groups did not differ. Further analysis was conducted to examine group effects on switching, words in a cluster, and cluster size. There was a significant group difference on switching $[\mathrm{F}(2,135)=24.0$, $p<.001]$ with the control group $(\mathrm{M}=8.0,95 \% \mathrm{CI}[7.1,8.8])$ differing from frontal $(\mathrm{M}=4.7,95 \% \mathrm{CI}[4.1,5.3])$ and non-frontal $(\mathrm{M}=5.3,95 \%$ CI $[4.6,5.9])$ groups, yet there was no difference between the two stroke groups. Finally, there was no statistically significant difference in any groups for words in a cluster $[\mathrm{F}(2,135)=0.49, p>.05]$ or cluster size $[\mathrm{F}(2,135)=10.6, p>.05]$.

We investigated the possibility that because stroke patients produced fewer responses in general, they did not have as many opportunities to make errors on the semantic and phonemic tasks. The ratio of errors to correct responses was compared between patients and controls. There was no statistically significant difference found between stroke patients and controls on the ratio of errors to correct responses on either phonemic task $(t(136)=1.02, p=0.31)$ or semantic task $(t$ $(136)=-0.56, p=0.58)$.

As previously mentioned, there was no significant difference in NIHSS scores between frontal and non-frontal groups. This indicated that overall stroke severity was similar across both groups. NIHSS score and performance on verbal fluency measures was evaluated (Table 3) to determine if fluency simply reflected stroke severity. Only the number of correct responses for animal fluency correlated significantly with stroke severity.

Table 3. Correlation between NIHSS and fluency measures for stroke group.

\begin{tabular}{|l|c|}
\hline Variable & NIHSS \\
\hline FAS Correct & -0.12 \\
\hline FAS Errors & 0.02 \\
\hline FAS Switches & -0.10 \\
\hline FAS Clusters & -0.14 \\
\hline FAS Cluster Size & 0.17 \\
\hline Animals Correct & $-0.27^{* *}$ \\
\hline Animals Errors & 0.01 \\
\hline Animals Switches & -0.15 \\
\hline Animals Clusters & -0.16 \\
\hline Animals Cluster Size & -0.03 \\
\hline
\end{tabular}

$* * p<.001 ; * p<.05$.

\section{Discussion}

This study investigated if performance on measures of verbal fluency (letter naming and animal naming tasks) was sensitive to lesion location and could differentiate between stroke patients with frontal vs. non-frontal damage. We also examined whether fluency was associated with age, education and stroke severity. The correlations between verbal fluency scores and age and education are consistent with previous findings in the literature $[2,20]$. The control group had more robust correlations between both age and education and letter and animal fluency performance. Age was negatively correlated with correct responses in letter fluency for the stroke group, but age correlated with both tasks for the control group. Education correlated with three measures of letter fluency for the controls, yet there was no correlation with education on either task for the stroke group. This suggests the cognitive impairments caused by a stroke eclipse the effects of age and education at the post-acute stage of recovery.

Compared to the controls, stroke patients in both groups showed similar performance patterns across the phonemic and semantic tasks. Differences in the number of correct responses and switching in semantic and phonemic tasks were consistent with our hypothesis that executive abilities are impaired in stroke patients, compared to healthy controls. The lack of a difference in the number of errors made by controls and patients in either letter or category fluency was unexpected. We surmise that patients were more inclined to withhold an incorrect response, rather than venturing a response that may be incorrect, thus producing less errors overall. This explanation supports a conceptual framework of execution function, as a collection of various processes with selective deficits in components of underlying executive skills. In the case of stroke, it appears that the ability to selfmonitor is intact, yet other facets like switching and clustering may be impaired [21].

Our hypothesis, based on previous imaging work, that frontal patients would produce more errors and exhibit poorer performance on switching in either phonemic or semantic tasks than non-frontal patients was not supported. Although both frontal and non-frontal groups performed more poorly on the phonemic task and semantic task compared to healthy controls, both groups produced similar scores on all quantitative and measures of letter and animal fluency. Frontal and non-frontal patients did not differ in the number of errors or switching in either phonemic or semantic tasks. This suggests that patients with lesions in the non-frontal regions are also susceptible to disruption in executive abilities. Although it is possible that a lack of clearly delineated homogenous groups (frontal vs. non-frontal) prevented us from detecting a difference, similarity in number of errors produced between frontal and non-frontal groups suggests that patients in both groups still self-monitor and withhold incorrect responses.

The similarity in scores and lack of discrimination on verbal fluency performance between the frontal and non-frontal groups is informative. Davidson et al. [22] used measures of verbal fluency, Trail Making Test, and Wisconsin Card Sorting Test to assess performance in participants with frontal region tumor resection; participants demonstrated poor performance in switching but not clustering, yet there was no lesion-specific relationship between performance on the measures. In another study, Tamez and colleagues [23] found similar findings in a stroke cohort with Trail Making and Digit Span Tests where both measures demonstrated sensitivity to brain damage, yet they lack specificity in being able to differentiate patients with frontal vs. non-frontal lesions. The similarity in performance across different 
lesions groups in acute setting on verbal fluency measures of executive function is significant. These findings suggest that neuropsychological assessments of verbal fluency (phonemic and semantic tasks) have strong utility in assessing impairments of executive abilities overall but contribute less to identifying specific lesion locations with particular performance deficits.

\section{Limitations}

Stroke lesions classification based on clinical imaging can be complicated due to CT vs. MRI sensitivity, age specificity and undocumented vascular damage among others. Despite the modest sample of stroke patients available, the lesions of very few participants fit the criteria for an exclusive group comprising solely of frontal lesions. One reason for this is that frontal lesions frequently result in aphasia, and therefore produce a group of necessarily excluded participants. This was reflected in the proportion and results that showed there were no statistically significant differences between patients with localized lesions in right, left and both hemispheres and performance on the fluency measures; potentially due to small sample sizes. As a result, the lesion diversity was limited. Only four of the frontal patients had lesions completely confined to the frontal regions. The remainder of the frontal group had lesions affecting other regions of the brain. Based on the lobar vascular classification we used, the postcentral gyrus (parietal lobe) was scored as frontal. The difficulty in placing stroke patients into a lesion category supports the use of techniques like Voxel-based Lesion-Symptom Mapping that eliminates broad lesion groupings. Another limitation concerns the acuity of when the stroke patients were assessed. The lesion data analyzed came from acute stroke patients, within 72 hours of admission. However, other studies with chronic stroke patients several months post-stroke, show similar results in the lack of lesion localization of neuropsychological tests $[21,24]$. Therefore, it is unlikely that the acute testing of verbal fluency contributed to the results. Conversely, the timing of these assessments in acute stroke care may serve to influence treatment planning and rehabilitation goals as they reveal deficists in performance in executive function. These cognitive deficits may be targeted during in-patient rehabilitation and addressed to improve stroke recovery.

\section{Disclosures}

The author does not have any disclosure to report.

\section{Acknowledgements}

The author would like to thank Mark Reddish and Drs. Lisa Connor, Desiree Head and Lucy Morris for this support on the conception of the study and critical feedback.

\section{References}

1. Lezak MD (2004) Neuropsychological Assessment 4e. Oxford University Press, USA: 518-522

2. Troyer A, Moscovitch M, Winocur G (1997) Clustering and switching as two components of verbal fluency: Evidence from younger and older healthy adults. Neuropsychology 11: 138-146. [Crossref]

3. Baldo JV, Schwartz S, Wilkins D, Dronkers NF (2006) Role of frontal versus temporal cortex in verbal fluency as revealed by voxel-based lesion symptom mapping. $J$ Int Neuropsychol Soc 12: 896-900. [Crossref]

4. Strauss EH (2006) A compendium of neuropsychological tests: Administration, norms and commentary. Oxford University Press 499-522

5. Mummery CJ, Patterson K, Hodges JR, Wise RJ (1996) Generating ‘tiger' as an animal name or a word beginning with T: differences in brain activation. Proc Biol Sci 263: 989-995. [Crossref]
6. Gourovitch ML, Kirkby BS, Goldberg TE, Weinberger DR, Gold JM, et al. (2000) A comparison of rCBF patterns during letter and semantic fluency. Neuropsychology 14: 353-360. [Crossref]

7. Troyer AK (2000) Normative data for clustering and switching on verbal fluency tasks J Clin Exp Neuropsychol 22: 370-378. [Crossref]

8. Tombaugh TN, Kozak J, Rees L (1999) Normative data stratified by age and education for two measures of verbal fluency: FAS and animal naming. Arch Clin Neuropsychol 14: 167-177. [Crossref]

9. Gladsjo JA, Schuman CC, Evans JD, Peavy GM, Miller SW, et al. (1999) Norms for letter and category fluency: demographic corrections for age, education, and ethnicity. Assessment 6: 47-178. [Crossref]

10. Crowe SF (1992) Dissociation of two frontal lobe syndromes by a test of verbal fluency J Clin Exp Neuropsychol 14: 327-339. [Crossref]

11. Ober BA, Dronkers NF, Koss E, Delis DC, Friedland RP (1986) Retrieval from semantic memory in Alzheimer-type dementia. J Clin Exp Neuropsychol 8: 75-92. [Crossref]

12. Troyer AK, Moscovitch M, Winocur G, Alexander MP, Stuss D (1998) Clustering and switching on verbal fluency: the effects of focal frontal- and temporal-lobe lesions. Neuropsychologia 36: 499-504. [Crossref]

13. Henry JD, Crawford JR (2004) A meta-analytic review of verbal fluency performance following focal cortical lesions. Neuropsychology 18: 284-295. [Crossref]

14. Schwartz S, Baldo J (2001) Distinct patterns of word retrieval in right and left frontal lobe patients: a multidimensional perspective. Neuropsychologia 39: 1209-1217. [Crossref]

15. Wolf TJ, Baum C, Conner LT (2009) Changing face of stroke: implications for occupational therapy practice. Am J Occup Ther 63: 621-625. [Crossref]

16. Damasio H, Damasio AR (1990) Lesion Analysis in Neuropsychology. J. Cognitive Neurosci 2: 156-157

17. Brott T, Adams HP, Olinger CP, Marler JR, Barsan WG, et al. (1989) Measurements of acute cerebral infarction: a clinical examination scale. Stroke 20: 864-870. [Crossref]

18. Adams HP, Davis PH, Leira EC, Chang KC, Bendixen BH, et al. (1999) Baseline NIH Stroke Scale score strongly predicts outcome after stroke A report of the Trial of Org 10172 in Acute Stroke Treatment (TOAST). Neurology 53: 126-126. [Crossref]

19. Schlegel D, Kolb SJ, Luciano JM, Tovar JM, Cucchiara BL, et al. (2003) Utility of the NIH Stroke Scale as a predictor of hospital disposition. Stroke 34: 134-137. [Crossref]

20. Elkins JS, Longstreth WT Jr, Manolio TA, Newman AB, Bhadelia RA, et al. (2006) Education and the cognitive decline associated with MRI-defined brain infarct. Neurology 67: 435-440. [Crossref]

21. McCabe DP, Roediger HL, McDaniel MA, Balota DA, Hambrick D.Z (2010) The relationship between working memory capacity and executive function: Evidence for a common executive attention construct. Neuropsychology 24: 222-243. [Crossref]

22. Davidson PS, Gao FQ, Mason WP, Winocur G, Anderson ND (2008) Verbal fluency, Trail Making, and Wisconsin Card Sorting Test performance following right frontal lobe tumor resection. J Clin Exp Neuropsychol 30: 18-32. [Crossref]

23. Tamez E, Myerson J, Morris L, White DA, Baum C, et al. (2011) Assessing executive abilities following acute stroke with the trail making test and digit span. Behav Neurol. 24: 177-185. [Crossref]

24. Leskelä M, Hietanen M, Kalska H, Ylikoski R, Pohjasvaara T, et al. (1999) Executive functions and speed of mental processing in elderly patients with frontal or nonfrontal ischemic stroke. Eur J Neurol 6: 653-661. [Crossref]

Copyright: (C)2016 Babulal GM. This is an open-access article distributed under the terms of the Creative Commons Attribution License, which permits unrestricted use, distribution, and reproduction in any medium, provided the original author and source are credited. 